\title{
A spotlight on redifferentiation strategies and target modulation in differentiated thyroid cancer
}

\author{
Désirée Deandreis $s^{1,8}$ (D) Petra Petranović Ovčariček ${ }^{2,8} \cdot$ Alfredo Campenni $^{3,8} \cdot$ Alexis Vrachimis $^{4,5,8}$. \\ Luca Giovanella ${ }^{6,7,8}$
}

Received: 30 April 2021 / Accepted: 5 July 2021 / Published online: 16 July 2021

(c) Italian Association of Nuclear Medicine and Molecular Imaging 2021

\section{Background}

Radioactive iodine (RAI) is a cornerstone for metastatic thyroid cancer treatment since the $40 \mathrm{~s}$ and the first real example of a theragnostic approach in Nuclear Medicine. The most important paradigm of the theragnostic approach is related to the possibility to identify by in vivo imaging the expression of a therapeutic target(s) as well as prognostic factors influencing patients' outcomes. In thyroid cancer, the target is the sodium-iodine symporter (NIS), whose expression on cell surface and functionality varies in different cancers. For the in vivo evaluation of NIS expression and subsequent NIS-targeted therapy several iodine isotopes can help us in obtaining diagnostic and dosimetric information $\left({ }^{123} \mathrm{I} ;{ }^{124} \mathrm{I}\right.$;

Désirée Deandreis

desiree.deandreis@unito.it

1 Department of Medical Sciences, Nuclear Medicine Division, AOU Città della Salute e della Scienza, University of Turin, C.so Dogliotti 14, 10126 Turin, Italy

2 Department of Oncology and Nuclear Medicine, University Hospital Center "Sestre Milosrdnice", Zagreb, Croatia

3 Unit of Nuclear Medicine, Department of Biomedical and Dental Sciences and Morpho-Functional Imaging, University of Messina, Messina, Italy

4 Department of Nuclear Medicine, German Oncology Center, University Hospital of the European University, Limassol, Cyprus

5 C.A.R.I.C. CANCER RESEARCH \& INNOVATION CENTER, Limassol, Cyprus

6 Clinic for Nuclear Medicine and Competence Centre for Thyroid Diseases, Imaging Institute of Southern Switzerland, Ente Ospedaliero Cantonale, Bellinzona, Switzerland

7 Clinic for Nuclear Medicine, University Hospital and University of Zurich, Zurich, Switzerland

8 European Association of Nuclear Medicine-Thyroid Committee, Vienna, Austria $\left.{ }^{131} \mathrm{I}\right)$ and treating thyroid cancer patients $\left({ }^{131} \mathrm{I}\right)$ [1]. A further step of the theragnostic approach is the possibility to pharmacologically manipulate target(s) expression to improve radionuclide therapy efficacy. In this field, thyroid cancer is again the first example of the feasibility of target(s) manipulation before ${ }^{131}$ I therapy to reinduce tumor differentiation. This paper has the principal aim to give a spotlight on principles and the available results of clinical trials focused on redifferentiation strategies in thyroid cancer, to share with the scientific community the need to implement research in this field as possible very promising direction for radionuclide therapy and to underline still open questions/perspectives for the future.

\section{Principles}

The clinical need for reinducing thyroid cancer differentiation arises from the fact that one-third of patients with metastatic thyroid cancer become refractory to radioactive iodine (RAI-R). The treatment of these patients is challenging as the second-line therapy is based on tyrosine kinase inhibitor administration, frequently reserved for patients with progressive RAI-R disease and a high tumor burden with several adverse events. In the era of personalized medicine, the possibility to manipulate and reinduce the expression of molecular targets for radionuclide therapy is fascinating and this approach could be applied early in the disease course to avoid toxicity and improve efficacy. Several compounds in the past (i.e. retinoic acid, PPAR- $\gamma$ inhibitors) have been investigated for redifferentiation approach in patients with metastatic RAI-R thyroid cancer but none demonstrated a clinically significant effect. More recently, in vitro studies have demonstrated that the activation of a specific intracellular pathway (i.e. Mitogen-Activated Protein Kinase or MAPK) involved in tumor proliferation and neoangiogenesis reduces the expression and activity of NIS with subsequent 
reduction of iodine avidity in thyroid cancer lesions. The activation of the MAPK pathway through MEK and ERK activation has been found in mutated tumors harboring BRAF, NRAS, or RET/PTC mutations. BRAF mutation is present in almost $50 \%$ of papillary thyroid cancers and may inhibit genes related to iodine metabolism, decrease NIS expression, and increase the risk of RAI-R disease. Furthermore, RET/PTC (papillary thyroid cancer) and RAS (follicular thyroid cancer) mutations are also related to the MAPK kinase pathway. According to pre-clinical data in mice, blockage of hyper-activated pathways could lead to a re-expression of NIS on the cell membrane [2,3].

\section{Clinical trials}

A summary of the available clinical studies is presented in Table 1.

The first in-human trial published in 2013, designed at Memorial Sloan Kettering Cancer Center (MSKCC, New
York, USA) on the basis of promising pre-clinical study in BRAF mutated mouse models, showed the potential of target therapy to reinduce a significant ${ }^{131}$ I uptake through the inhibition of MAPK cascade in patients treated by selumetinib (MEK inhibitor) before ${ }^{131}$ I treatment [4]. Patients with refractory thyroid cancer without ${ }^{131} \mathrm{I}$ uptake, patients with persistent or progressive disease despite previous ${ }^{131}$ I treatment, or patients with at least one ${ }^{18} \mathrm{~F}$-FDG avid lesions were included. All patients underwent ${ }^{124}$ I PET/CT after rhTSH administration and were subsequently treated for 4 weeks with target therapy. A further ${ }^{124}$ I PET/CT after rhTSH administration was then performed to evaluate the iodine uptake restoration and to perform lesion-based dosimetry. When calculated activities required to achieve a presumed absorbed dose $\geq 2000 \mathrm{cGy}$ in the metastatic lesions were $\leq 11.1 \mathrm{GBq}$, patients were treated with a personalized ${ }^{131}$ I activity after recombinant human rhTSH administration. Radioactive iodine uptake was restored in 12/20 included patients and finally, 8/12 patients were treated with 5 partial responses and 3 stable diseases at 6 months follow up.

Table 1 Summary of published clinical trials

\begin{tabular}{|c|c|c|c|c|c|c|c|c|c|}
\hline & Study type & $\begin{array}{l}N \text { of } \\
\text { patients }\end{array}$ & Mutation & $\begin{array}{l}\text { Pathway } \\
\text { Mechanism }\end{array}$ & Drug & $\begin{array}{l}\text { Duration of } \\
\text { TKI before } \\
\text { RAI }\end{array}$ & Methods & $\begin{array}{l}N \text { of patients } \\
\text { with rein- } \\
\text { duced I131 } \\
\text { uptake and } \\
\text { treated with } \\
\text { RAI }\end{array}$ & $\begin{array}{l}\text { Response } \\
\text { according to } \\
\text { RECIST } 1.1\end{array}$ \\
\hline $\begin{array}{c}\text { Ho et al. } \\
2013 \\
\text { NEJM }\end{array}$ & Clinical trial & 20 & $\begin{array}{l}\text { BRAF, } \\
\text { NRAS, } \\
\text { RET/PTC, } \\
\text { WT }\end{array}$ & MEK & Selumetinib & 4 weeks & ${ }^{124} \mathrm{I}$ PET/CT & $8 / 20$ & $\begin{array}{l}5 \mathrm{PR} \text { and } 3 \\
\mathrm{SD}\end{array}$ \\
\hline $\begin{array}{l}\text { Rothenberg } \\
\text { et al. } 2015 \\
\text { Clinical } \\
\text { Cancer } \\
\text { Research }\end{array}$ & Clinical trial & 10 & $\begin{array}{l}\text { BRAF } \\
\text { V600-E }\end{array}$ & BRAF & Dabrafenib & 25 days & $\begin{array}{l}{ }^{131} \text { I diagnos- } \\
\text { tic WBS }\end{array}$ & $6 / 10$ & $\begin{array}{l}2 \mathrm{PR} \text { and } 4 \\
\text { SD }\end{array}$ \\
\hline $\begin{array}{l}\text { Dunn et al. } \\
2019 \\
\text { JCEM }\end{array}$ & Clinical trial & 10 & $\begin{array}{l}\text { BRAF } \\
\text { V600-E }\end{array}$ & BRAF & Vemurafenib & 4 weeks & ${ }^{124} \mathrm{I}$ PET/CT & $4 / 10$ & $\begin{array}{l}2 \mathrm{PR} \text { and } 2 \\
\text { SD }\end{array}$ \\
\hline $\begin{array}{l}\text { Jaber et al. } \\
2018 \\
\text { JCEM }\end{array}$ & $\begin{array}{l}\text { Retrospec- } \\
\text { tive }\end{array}$ & 13 & $\begin{array}{l}\text { BRAF, } \\
\text { NRAS/ } \\
\text { KRAS, } \\
\text { WT }\end{array}$ & $\begin{array}{l}\text { BRAF or } \\
\text { MEK }\end{array}$ & $\begin{array}{l}\text { Dabrafenib } \\
\text { or } \\
\text { Vemurafenib } \\
\text { or } \\
\text { Dabrafenib } \\
\text { and } \\
\text { trametinib } \\
\text { or } \\
\text { Trametinib } \\
\text { or } \\
\text { Investiga- } \\
\text { tional drug }\end{array}$ & $\begin{array}{l}\text { Median } \\
14.3 \text { months } \\
\quad(1-76.4)\end{array}$ & $\begin{array}{l}{ }^{131} \text { I diagnos- } \\
\text { tic WBS }\end{array}$ & $9 / 13$ & $\begin{array}{l}3 \mathrm{PR} \text { and } 6 \\
\text { SD }\end{array}$ \\
\hline $\begin{array}{l}\text { Leboulleux } \\
\text { et al. } 2019 \\
\text { Thyroid }\end{array}$ & Case report & 1 & $\begin{array}{l}\text { BRAF } \\
\text { K601E }\end{array}$ & $\begin{array}{l}\text { BRAF and } \\
\text { MEK }\end{array}$ & $\begin{array}{r}\text { Dabrafenib- } \\
\text { trametinib }\end{array}$ & 2 months & $\begin{array}{l}{ }^{131} \text { I diagnos- } \\
\text { tic WBS }\end{array}$ & $\begin{array}{l}1 / 1 \\
\text { Not treated } \\
\quad \text { with }{ }^{131} \mathrm{I}\end{array}$ & $\begin{array}{l}1 \mathrm{SD} \\
\text { With drugs } \\
\text { combina- } \\
\text { tion alone }\end{array}$ \\
\hline
\end{tabular}

TKI Tyrosine kinase inhibitor, RAI Radioactive Iodine, $P R$ Partial Response, SD Stable Disease, WBS Whole body scan 
Nevertheless, RAI uptake reinduction was observed in only 4/9 patients harboring BRAF mutation and only 1 patient among them was treated with ${ }^{131}$ I and achieved an objective response. On the other hand, 5/5 patients with NRAS mutation showed reinduction of RAI uptake and all were treated with ${ }^{131}$ I with an objective response. The remaining 2 treated patients had a wild type and RET/PTC mutated thyroid carcinoma, respectively. The relationship between the reinduction of ${ }^{131} \mathrm{I}$ uptake and the mutational status (BRAF, RAS, RET/PTC, TERT) of thyroid cancer is challenging and needs further investigation. In the MSKCC trial, the low efficacy of redifferentiation strategy by selumetinib in BRAF mutated tumors was mainly related to a sub-optimal inhibition of the MAPK pathway. Consequently, specific BRAF inhibitors (i.e. dabrafenib, vemurafenib, or trametinib) may induce a sustained MAPK pathway inhibition and have been tested, as single agents or in combination, in re-differentiation clinical trials in BRAF mutated tumors. The available studies showed reinduction of ${ }^{131} \mathrm{I}$ uptake in around $50 \%$ of patients and achievement of objective response or disease stabilization after ${ }^{131}$ I treatment especially in cases in which pretherapeutic dosimetric evaluation has been applied and demonstrated a sufficient absorbed dose within target lesions. The study from Rothenberg et al. tested 4 weeks of treatment with dabrafenib in 10 patients harboring BRAFV600 mutation and with an absence of ${ }^{131}$ I uptake at whole-body scan within 14 months before the inclusion in the trial [5]. Six of the 10 patients showed new ${ }^{131}$ I uptake foci and were treated consequently with radioactive iodine associated to a prolongation of 2 weeks of dabrafenib treatment, achieving 2 partial responses and 4 stable diseases at 3 months radiological restaging evaluation. In this study, the reappearance of ${ }^{131}$-I avidity was evaluated by a pre-therapeutic diagnostic ${ }^{131}$ I whole-body scan (WBS) after rhTSH stimulation and patients with new iodine uptake, not necessarily present in all the known lesions, were treated with a fixed activity of 5.5 GBq of radioactive iodine without dosimetry evaluation. Dunn et al. study from MSKCC applied the same inclusion criteria of the selumetinib trial and tested 4 weeks of treatment with vemurafenib, an ATP-competitive BRAF inhibitor, in 10 patients harboring BRAFV600 mutation [6]. Six patients presented uptake reinduction at ${ }^{124}$ I PET/CT, but only four met the lesional dosimetry threshold according to the same protocol applied in the first MSKCC trial and were treated with ${ }^{131} \mathrm{I}$. All four patients did not present disease progression at 6 months follow up, two of them achieving a partial response and two stable diseases, respectively. Interestingly the available data from molecular studies obtained by tumor biopsy in 3 patients showed a "reprogrammation" of the genomic signature after a short-time target therapy with an increase of the so called "Thyroid Differentiation score" according to the Cancer Genome Atlas TCGA classification, a decrease in the mitotic index and increase in the
NIS mRNA. These data confirm a realistic effect of the drug on the molecular profile and the reinduction of the expression of genes involved in iodine uptake especially in a more differentiated tumor at baseline. Jaber et al. study retrospectively evaluated 13 patients with RAI-R thyroid cancer treated with single agent or MEK/BRAF inhibitors combination that underwent a ${ }^{131}$ I diagnostic whole-body scan during the treatment period [7]. Nine patients harbored BRAF V600E and 3 NRAS mutations, respectively. The remaining case was a Wild Type (WT) thyroid cancer. The median duration of target therapy before the diagnostic WBS was 14.3 months (range 0.9-76.4). Among the included patients, 8 showed a restoration of ${ }^{131} \mathrm{I}$ uptake and were treated with a median activity of $7.4 \mathrm{GBq}$ of radioactive iodine. A further patient was empirically treated despite the absence of clear evidence of ${ }^{131} \mathrm{I}$ avidity restoration. In only 3 patients a dosimetric pre-therapeutic evaluation has been performed, while in the remaining cases an empiric activity of ${ }^{131} \mathrm{I}$ was administrated. Before ${ }^{131}$ I treatment 8 patients had stable disease and 1 patient progressive disease. After ${ }^{131}$ I treatment and a median follow up of 14.3 months, 3 patients achieved a partial response while 6 patients presented a stable control of disease according to radiological evaluation, including the patient empirically treated.

Other clinical trials are ongoing (EudraCT No 2015-002, 269-47, NCT03244956 NCT04554680, NCT04619316) and preliminary data seem to support better results in terms of objective response using the combination of different drugs targeting MEK and BRAF to induce a more robust MAPK pathway inhibition [8].

\section{Perspectives and conclusions}

Despite very promising perspectives, there are still several open questions to define the best approach.

In particular, there is still few evidence about the best candidate for this approach, the most appropriate therapy sequence and drug combination, the role of a direct effect of short-term target therapy alone on tumor response, molecular pre-treatment schedule, ${ }^{131} \mathrm{I}$ activity to be administered, and the most reliable tools to evaluate response accurately. First, the subset of patients that could benefit from this approach achieving tumor regression or progression free survival improvement is under debate. Patients included in the first pilot studies are heterogeneous accounting for patients with RAI-R tumors without ${ }^{131}$ I uptake and/or with progressive disease despite previous ${ }^{131}$ I therapy and/or ${ }^{18} \mathrm{~F}$-FDG PET positive lesions. Nevertheless, patients with RAI-R but with slowly progressive disease, without extensive tumor burden, and with a partially preserved pattern of differentiation are likely the best candidates to receive available redifferentiation therapies. This may avoid, on one side, long 
lasting TKI treatment with the risk of adverse events and, on the other side, enhance tumor control in an early phase of the disease. Across the studies, these drugs have been in most cases administrated for a short period ranging from 3-4 weeks before ${ }^{131}$ I treatment with a low incidence of toxicity, making this approach safe and well-tolerated. Furthermore, some cases of direct anti-tumoral action of the drug itself before ${ }^{131}$ I treatment have been reported, suggesting a double effect on disease response. In Dunn et al. study for example vemurafenib alone resulted in tumor regression within 6 weeks after completing vemurafenib and before ${ }^{131} \mathrm{I}$ treatment in 7 patients, including the 4 patients that showed reinduction of iodine uptake and 3 more patients among the cases not treated with radioactive iodine [6]. Second, the direct effect of these drugs on iodine uptake restoration can be accurately evaluated before treatment by ${ }^{124}$ I PET/CT, or as an alternative by ${ }^{123} \mathrm{I}$ or ${ }^{131} \mathrm{I}$ whole-body imaging. ${ }^{124} \mathrm{I}$ PET-CT has the advantage over traditional ${ }^{131}$ I diagnostic whole-body scan to quantify the iodine uptake through semiquantitative analysis even if there is not a standardized methodology or cut off proposed, while applying more traditional technique may lead to underestimation of iodine uptake restoration. Nevertheless, the reinduction of iodine uptake is not sufficient per se' to define a patient as "newly" responsive to ${ }^{131}$ I therapy and dosimetry-based approach is crucial to evaluate if the delivered absorbed dose to the lesions is sufficient to induce a therapeutic effect and to determine the most appropriate ${ }^{131}$ I activity. ${ }^{124}$ I PET/CT could be the technique of choice also for this purpose but unfortunately, it is not widely available and at this moment it is employed for clinical trials only. ${ }^{131}$ I based dosimetry could be feasible but the risk of stunning is still present even if low tracer activity is administered.

Third, the best modality to evaluate the response after ${ }^{131}$ I treatment and the duration of response during a time without further systemic treatments should be investigated. In clinical trials, RECIST 1.1 criteria are the most widely used criteria to evaluate morphological response at 3 or 6 months but it is not always correlated with a decrease of thyroglobulin levels. On one side thyroglobulin cannot be the best marker to evaluate the response in case of poorly differentiated histological subtype and, interestingly, on the other side some cases of increase of tumoral marker levels in presence of morphological response have been described and probably related to the redifferentiation process with re-expression of tumoral markers compared to baseline levels. Some cases of long lasting and durable response after the last ${ }^{131}$ I treatment have been reported but longer follow up is needed to confirm this data as well as data on the impact of this approach on progression free survival and overall survival are lacking. Finally, several other targets and pathways for the re-differentiation approach are under investigation in pre-clinical studies such as AKT, PI3K, and the NOTCH signaling pathways and seem to be promising for future applications [9].

In conclusion, even if further studies and comparative trials are needed to confirm the clinical usefulness of combining molecular redifferentiating drugs and ${ }^{131} \mathrm{I}$ in patients with RAI-R thyroid cancers, this approach represents a step forward in the theragnostic paradigm and a promising option for thyroid cancer patients.

\section{Declarations}

Conflict of interest The authors have nothing to declare. This article does not contain any studies with human or animal subjects performed by any of the authors.

\section{References}

1. Nagarajah J, Janssen M, Hetkamp P, Jentzen W (2017) Iodine symporter targeting with 124I/131I theranostics. J Nucl Med 58:34S-38S. https://doi.org/10.2967/jnumed.116.186866

2. Chakravarty D, Santos E, Ryder M et al (2011) Small-molecule MAPK inhibitors restore radioiodine incorporation in mouse thyroid cancers with conditional BRAF activation. J Clin Invest 121(12):4700-4711. https://doi.org/10.1172/JCI46382

3. Nagarajah J, Le M, Knauf JA et al (2016) Sustained ERK inhibition maximizes responses of BrafV600E thyroid cancers to radioiodine. J Clin Invest 126(11):4119-4124. https://doi.org/10.1172/ JCI89067

4. Ho AL, Grewal RK, Leboeuf R et al (2013) Selumetinib enhanced radioiodine uptake in advanced thyroid cancer. $\mathrm{N}$ Engl $\mathrm{J}$ Med 368:623-632

5. Rothenberg M, McFadden DG, Palmer EL, Daniels GH, Wirth LJ (2015) Redifferentiation of iodine-refractory BRAF V600Emutant metastatic papillary thyroid cancer with dabrafenib. Clin Cancer Res 21(5):1028-1035. https://doi.org/10.1158/1078-0432. CCR-14-2915

6. Dunn LA, Sherman EJ, Baxi SS et al (2019) Vemurafenib redifferentiation of BRAF mutant, RAI-refractory thyroid cancers. J Clin Endocrinol Metab 104:1417-1428

7. Jaber T, Waguespack SG, Cabanillas ME et al (2018) Targeted therapy in advanced thyroid cancer to resensitize tumors to radioactive iodine. J Clin Endocrinol Metab 103(10):3698-3705. https://doi.org/10.1210/jc.2018-00612

8. Leboulleux S, Dupuy C, Lacroix L et al (2019) Redifferentiation of a BRAF K601E-mutated poorly differentiated thyroid cancer patient with dabrafenib and trametinib treatment. Thyroid 29(5):735-742. https://doi.org/10.1089/thy.2018.0457

9. Buffet C, Wassermann J, Hecht F et al (2020) Redifferentiation of radioiodine-refractory thyroid cancers. Endocr Relat Cancer 27(5):R113-R132

Publisher's Note Springer Nature remains neutral with regard to jurisdictional claims in published maps and institutional affiliations. 